\title{
Anterior Chamber Retained Lens Fragments After Cataract Surgery: A Case Series and Narrative Review
}

\author{
Majid Moshirfar (iD) ${ }^{1-3}$ \\ Adam L Lewis ${ }^{4}$ \\ James $\mathrm{H}$ Ellis $^{5}$ \\ Shannon E McCabe (D) 1,6 \\ Yasmyne C Ronquillo (D) \\ Phillip C Hoopes Sr (D) \\ 'Hoopes Vision Research Center, \\ Hoopes Vision, Draper, UT, USA; 'John \\ A. Moran Eye Center, Department of \\ Ophthalmology and Visual Sciences, \\ University of Utah School of Medicine, \\ Salt Lake City, UT, USA; ${ }^{3}$ Utah Lions Eye \\ Bank, Murray, UT, USA; ${ }^{4}$ Kansas City \\ University, Kansas City, MO, USA; \\ ${ }^{5}$ University of Utah School of Medicine, \\ Salt Lake City, UT, USA; ${ }^{6}$ Mission Hills \\ Eye Center, Pleasant Hill, CA, USA
}

Introduction: Retained lens fragments in the anterior chamber following cataract extraction (CE) with phacoemulsification are rare but can lead to significant patient morbidity. Our study aimed to identify risk factors associated with retained lens fragments.

Methods: Patients who underwent cataract surgery and subsequently identified to have retained lens fragments in the anterior segment were included. Incidence per year, patient demographics, visual acuity, ocular biometrics, surgical technique, surgeon performing CE, and outcomes were collected retrospectively and compared to a control group.

Results: Twenty-four patients were identified with retained lens fragments, with an incidence of $0.10 \%$. The mean age was 76 years $\pm 6.72(60-80)$ compared to $63 \pm 11.41(22-86)$ in the control group ( $p<0.001$ ). Patients with UDVA 20/150 or worse experienced a greater average improvement in visual acuity compared to patients with UDVA better than 20/150 ( $\operatorname{logMAR} 0.46$ vs $\log$ MAR 0.05 ). The mean intraocular pressures before (CE), after CE but before fragment removal, and following fragment removal were $14 \mathrm{mmHg} \pm 2.59,19 \mathrm{mmHg}$ \pm 8.20 , and $11 \mathrm{mmHg} \pm 2.75$, respectively. Twenty-two patients presented with inferiorly located fragments. Statistically significant biometrics include mean anterior chamber depth ( $3.1 \mathrm{~mm} \pm 0.37$ vs $3.33 \mathrm{~mm} \pm 0.39, \mathrm{p}=0.01)$ and lens thickness $(4.77 \mathrm{~mm} \pm 0.44$ vs $4.35 \mathrm{~mm}$ $\pm 0.44, \mathrm{p}=<0.001)$. Yearly incidence rates per surgeon ranged from $0.00 \%$ to $0.85 \%$. In 2003 and 2004, one surgeon had significantly higher incidence rates $(0.31$ and $0.40 \%)$ compared to the average combined rate of all surgeons throughout the study $(0.10)$, with $p$ values of 0.001 and 0.003 , respectively. The mean number of days between $\mathrm{CE}$ and fragment removal was 26 \pm 40 (1-138).

Conclusion: Increased patient age, shallow anterior chamber depth, and thick lens may be risk factors for retained lens fragments. There may be additional surgeon-specific risk factors. Phacoemulsification technique (Divide-and-Conquer versus Horizontal Chop) showed no significant difference.

Keywords: retained lens fragments, phacoemulsification, cataract surgery, complications of cataract surgery, refractory corneal edema

\section{Introduction}

Cataract surgery is one of the most commonly performed surgeries in the world. ${ }^{1}$ A rare but important complication of cataract surgery is the retention of lens fragments. ${ }^{2}$ This occurs following phacoemulsification when small fragments of the lens remain in the anterior or posterior chambers, within the lens capsular bag, or in the vitreous. Although the incidence of retained lens fragments is only $0.1 \%$ -
Correspondence: Majid Moshirfar Medical Director Hoopes Vision Research Center, Hoopes Vision, II820 S. State Street Suite \#200, Draper, UT, 84020, USA

Tel + I 80I-568-0200

$\mathrm{Fax}+1$ 80I-563-0200

Email cornea2020@me.com 
$1.5 \%,^{3-5}$ this complication may lead to significant patient morbidity including decreased visual acuity, corneal edema, glaucoma, retinal detachment, and cystoid macular edema. ${ }^{6,7}$ In addition, fragment retention commonly requires surgical intervention as symptoms are frequently refractory to standard medical management. ${ }^{8}$ Most cases of retained lens fragments are identified post-op day 1 , but the timeline for identification is variable with some reports of identification and diagnosis years later. ${ }^{9}$

The focus of this study is to evaluate patients with retained fragments in the anterior chamber following cataract surgery with phacoemulsification. As the number of cataract surgeries continues to rise, there is a greater need to evaluate why some patients may be more prone to lens fragment retention than others. The limited existing scientific literature regarding retained lens fragments in the anterior chamber following uneventful cataract surgery with phacoemulsification prompts further study and analysis. ${ }^{6}$ This study analyzes patient demographics, ocular biometrics, surgical technique, and outcomes. Such findings may help guide patient management and provide physicians important insight on patient risk factors to ensure the highest quality of care.

\section{Methods}

This study is a retrospective analysis of 24 patients who underwent cataract surgery with phacoemulsification and were subsequently identified to have unilateral retention of lens fragments in the anterior segment between April 2003 and March 2021. Patients with lens fragments falling into the vitreous chamber were excluded from this study. Incidence rates were calculated yearly by dividing the number of retained lens fragment patients by the total number of cataract surgeries performed in that year. Patients were selected from our surgical database and deidentified. All surgeries were performed by five surgeons using either a Horizontal Chop or Divide-and-Conquer phacoemulsification technique. All patients were diagnosed with retained lens fragments using slit lamp examination following cataract surgery. Patients gave informed consent for all procedures and the use of their clinical data in research. This study was approved by the Hoopes Vision Ethics Committee (Draper, UT) and Brany Institutional Review Board (New Hyde Park, NY).

After informed consent was obtained, fragment removal procedures were done in the operating room under sterile conditions. The original clear corneal incision was reopened using a cannula or a keratome, if necessary.
Viscoelastic and/or balanced saline solution on a cannula was used to flush the fragment out of the main incision. If this technique was unsuccessful, the irrigation and aspiration handpiece was used to remove the fragment under low flow settings. The wound was hydrated and confirmed to be watertight. Patients were instructed to use third- or fourth-generation fluoroquinolone antibiotic eye drops four times daily for one week. Patients were also started on a topical steroid medication four times daily and tapered weekly over one month.

Patient demographics, visual acuity, ocular biometrics, surgical technique, and outcomes were collected from patient charts. Incidence rates were analyzed using chisquare tests. For comparison, we used a control group of 117 patients (233 eyes) that underwent uncomplicated cataract surgery between January 1st, 2019 - July 1st, 2020. Axial length (AL), anterior chamber depth (ACD), aqueous depth (AD), lens thickness (LT), keratometry, and white-to-white values were obtained with optical biometry. Intraocular pressure was measured with Goldmann applanation tonometry. Patient characteristics and biometrics were compared to the control group and analyzed using a two-tail $t$-test. Results were evaluated, and statistical significance was defined as a $\mathrm{p}$ value of less than 0.05 .

\section{Results}

This study included 24 patients who underwent cataract surgery with phacoemulsification and were subsequently diagnosed and treated for unilateral retention of lens fragments in the anterior chamber. During the study, 23,232 lens removal surgeries were performed, making the total incidence of retained fragments $0.10 \%$. Patient characteristics and management data are summarized in Table 1. The mean age of patients with retained lens fragments was statistically older than our control group (76 years \pm 6.7 vs 63 years $\pm 11.41, \mathrm{p}<0.001)$ with 12 females and 12 males. According to the Lens Opacities Classification System, ${ }^{10}$ thirteen patients presented with $2+$ nuclear sclerotic (NS) cataract, four presented with $3+\mathrm{NS}$ cataract, six presented with $2+\mathrm{NS} / 2+$ cortical cataract $(\mathrm{CC})$, and one presented with a $4+$ cortical cataract.

Following $\mathrm{CE}$ and upon presentation with lens fragments, $8 \%$ of patients had an uncorrected distance visual acuity (UDVA) of 20/20 or better, 54\% had a UDVA of 20/ 40 or better, $71 \%$ had a UDVA of $20 / 100$ or better, and $92 \%$ had a UDVA of 20/200 or better. On post-op day 1 following fragment removal, $13 \%$ of patients had a UDVA of $20 / 20$ or better, $63 \%$ had a UDVA of $20 / 40$ or better, 


\begin{tabular}{|c|c|c|c|c|c|c|c|c|c|c|c|c|c|c|}
\hline 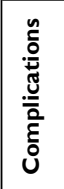 & $\begin{array}{l}\stackrel{0}{\tilde{0}} \\
\text { ż }\end{array}$ & $\begin{array}{l}\stackrel{0}{0} \\
\text { ż }\end{array}$ & $\begin{array}{l}\text { : } \\
\stackrel{0}{0} \\
\text { L }\end{array}$ & 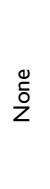 & $\begin{array}{l}\stackrel{0}{\tilde{D}} \\
\stackrel{5}{Z}\end{array}$ & $\begin{array}{l}\stackrel{0}{0} \\
\text { zo }\end{array}$ & 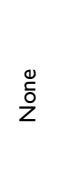 & $\begin{array}{l}\text { 号 } \\
\text { zo }\end{array}$ & $\begin{array}{l}\frac{0}{5} \\
z \\
\end{array}$ & $\begin{array}{l}\frac{0}{5} \\
\frac{5}{z}\end{array}$ & 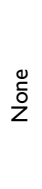 & 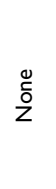 & 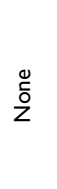 & 岂 \\
\hline
\end{tabular}

芯苔

\begin{tabular}{|c|c|c|c|c|c|c|c|}
\hline 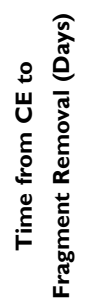 & \pm & $\infty$ & \pm & $\sigma$ & & $\stackrel{\infty}{\underline{m}}$ & - \\
\hline$\ddot{E}=$ & & & & & & & \\
\hline
\end{tabular}

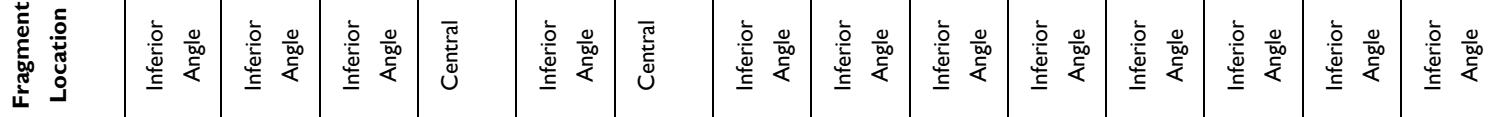

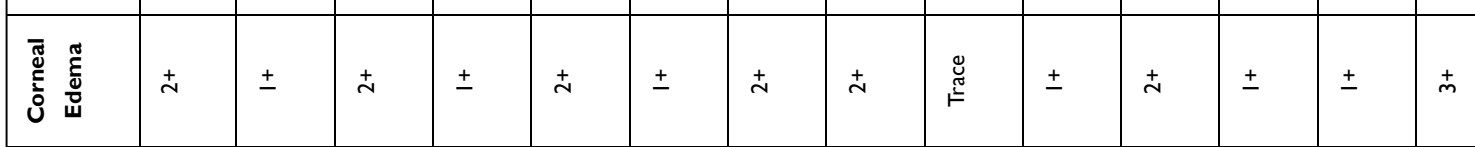

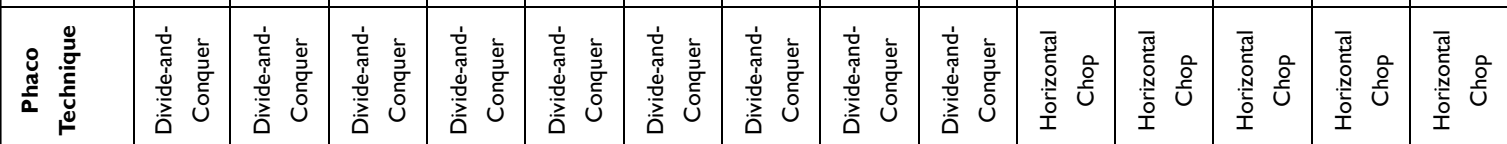

\begin{tabular}{|c|c|c|c|c|c|c|c|c|c|c|c|c|c|c|}
\hline 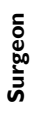 & - & - & - & - & $\sim$ & $\sim$ & $\sim$ & $m$ & $\sim$ & $\sigma$ & $\sigma$ & $\sigma$ & $\sigma$ & $\nabla$ \\
\hline$\underline{\underline{\xi}}$ & 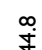 & $\stackrel{ \pm}{ \pm}$ & & $\overline{\dot{n}}$ & مُّ & 0 & † & 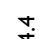 & - & & & & $\circ$ & 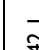 \\
\hline
\end{tabular}

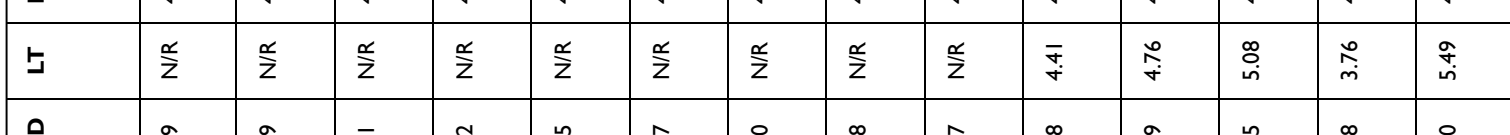

\begin{tabular}{|c|c|c|c|c|c|c|c|c|c|c|c|c|c|c|}
\hline$\stackrel{q}{q}$ & م્ & 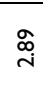 & $\underset{\dot{m}}{\bar{f}}$ & $\underset{\sim}{\tilde{m}}$ & $\underset{m}{q}$ & $\stackrel{\bar{m}}{\bar{m}}$ & $\stackrel{\text { }}{\text { j}}$ & 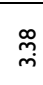 & 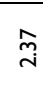 & 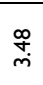 & iे & $\stackrel{\stackrel{h}{n}}{\text { n. }}$ & 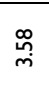 & 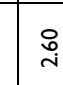 \\
\hline ــ & స్తి & $\stackrel{\sim}{\sim}$ & $\tilde{m}$ & $\bar{\sigma}$ & ণุ & oి & ర్ & f & $\overline{\underline{m}}$ & 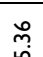 & $\stackrel{\sim}{\sim}$ & $\stackrel{ㅇ}{\dot{m}}$ & $\stackrel{\mathscr{m}}{m}$ & $\stackrel{\infty}{\infty}$ \\
\hline
\end{tabular}

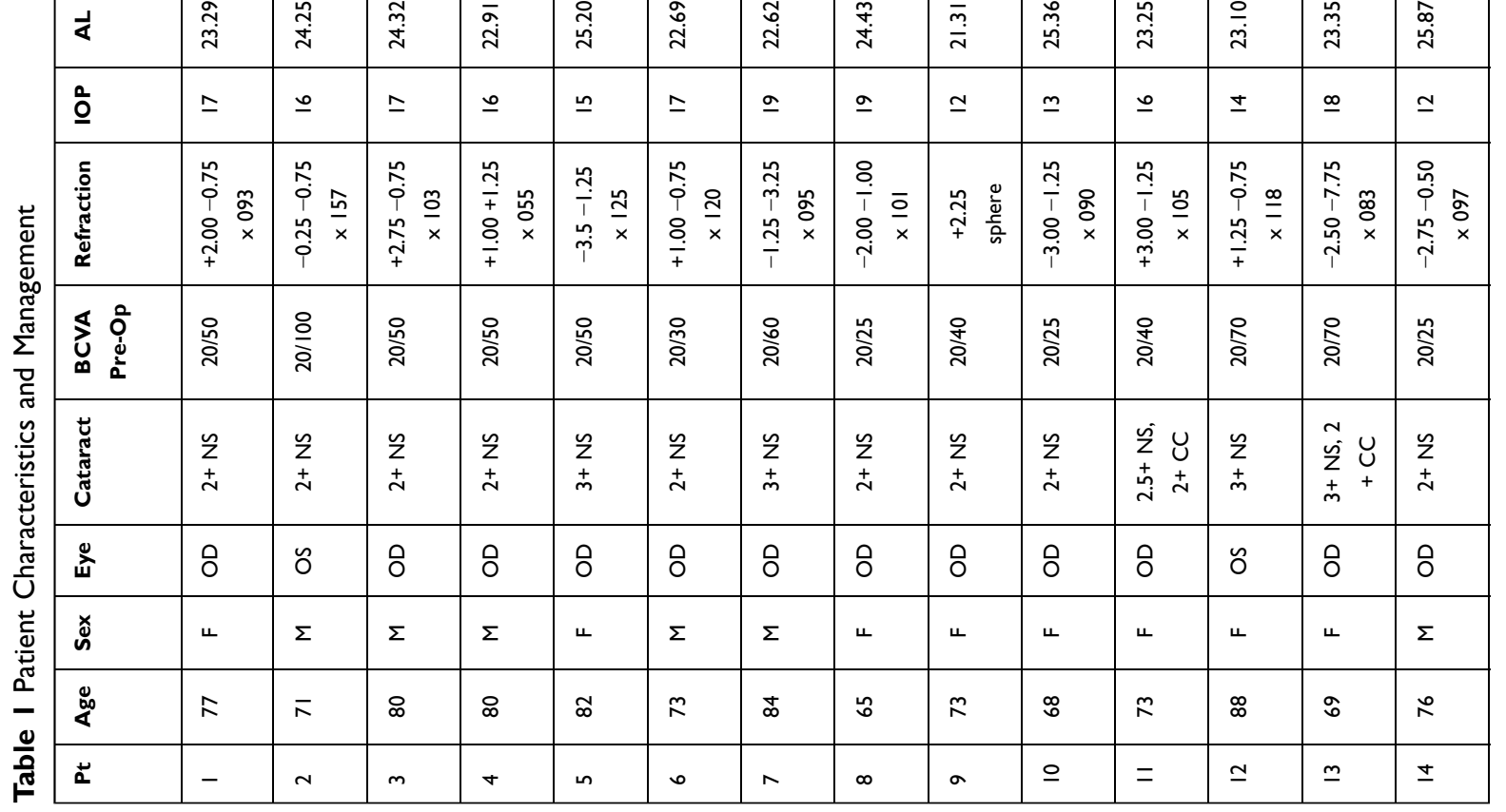




\begin{tabular}{|c|c|c|c|c|c|c|c|c|c|c|c|}
\hline 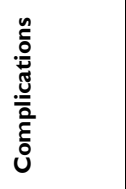 & 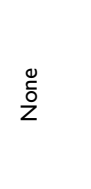 & 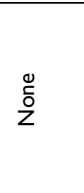 & $\begin{array}{l}\frac{0}{0} \\
\stackrel{0}{z}\end{array}$ & $\begin{array}{l}\frac{0}{5} \\
\frac{0}{2}\end{array}$ & $\begin{array}{l}\frac{0}{0} \\
\stackrel{0}{z}\end{array}$ & $\begin{array}{l}\frac{0}{0} \\
\frac{0}{z}\end{array}$ & $\sum_{\text {U }}^{山}$ & $\begin{array}{l}\frac{0}{\tilde{0}} \\
\frac{0}{2}\end{array}$ & $\begin{array}{l}\frac{0}{0} \\
\frac{0}{2}\end{array}$ & $\stackrel{0}{\frac{0}{0}}$ & 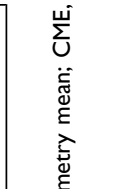 \\
\hline 芯蓠 。̊ & 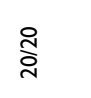 & ڤั่ & ঙั่ & 商 & ڤั่ & $\stackrel{n}{\grave{n}}$ & ণัָे & 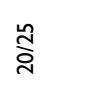 & ঙัָे & 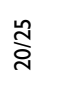 & $\ddot{\xi}$ \\
\hline 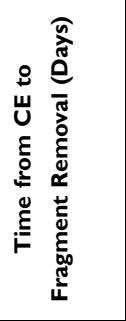 & - & $\bar{N}$ & $\bar{\sim}$ & $\stackrel{m}{m}$ & $m$ & - & $r$ & - & - & $\tilde{\underline{m}}$ & 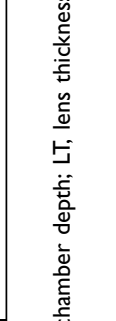 \\
\hline 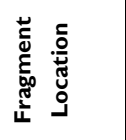 & 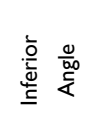 & 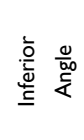 & 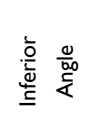 & 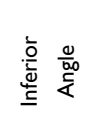 & 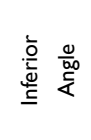 & 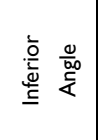 & 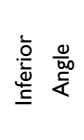 & 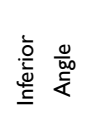 & 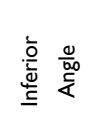 & 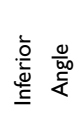 & 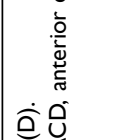 \\
\hline 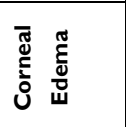 & $\underset{\mathscr{E}}{\stackrel{\mathscr{g}}{E}}$ & 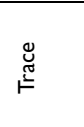 & \pm & \pm & \pm & \pm & $\stackrel{ \pm}{\sim}$ & $\stackrel{+}{\sim}$ & \pm & $\stackrel{+}{\stackrel{+}{m}}$ & \\
\hline 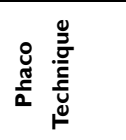 & 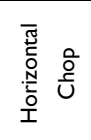 & 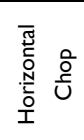 & 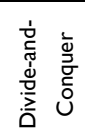 & 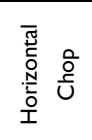 & 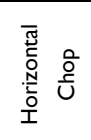 & 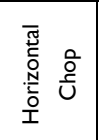 & 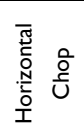 & 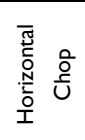 & 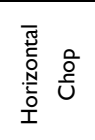 & 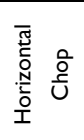 & 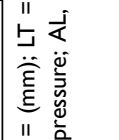 \\
\hline 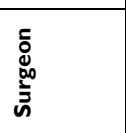 & $\sigma$ & $\sigma$ & in & $\sigma$ & $\sigma$ & $\sigma$ & $\sigma$ & $\sigma$ & + & $\sigma$ & 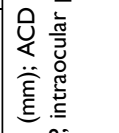 \\
\hline$\underline{\xi}$ & $\tilde{\Im}$ & $\stackrel{⿱ 亠 乂}{+}$ & $\stackrel{n}{\mathfrak{q}}$ & $\stackrel{m}{\tilde{q}}$ & $\stackrel{\sim}{\tilde{\gamma}}$ & $\tilde{\text { qु }}$ & $\overline{\mathcal{g}}$ & $\stackrel{\infty}{\tilde{\gamma}}$ & $\stackrel{\circ}{\stackrel{9}{f}}$ & $\overline{\dot{q}}$ & \\
\hline$\Xi$ & ì & $\stackrel{\stackrel{p}{q}}{\dot{r}}$ & 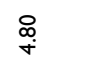 & $\underset{\dot{q}}{\stackrel{q}{f}}$ & $\underset{+\infty}{\infty}$ & $\stackrel{o}{z}$ & $\stackrel{\frac{\alpha}{z}}{z}$ & $\overline{\text { in }}$ & 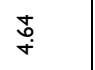 & $\underset{\alpha}{\alpha}$ & \\
\hline O̊ & స్ & $\stackrel{\circ}{\check{m}}$ & $\overline{\bar{m}}$ & $\underset{\text { mi }}{\text { in }}$ & $\underset{\sim}{\tilde{m}}$ & $\stackrel{\leftrightarrow}{i}$ & $\stackrel{\stackrel{\sim}{m}}{m}$ & $\underset{i}{\stackrel{N}{i}}$ & $\frac{N}{m}$ & $\underset{j}{+}$ & \\
\hline$\frac{1}{\varangle}$ & 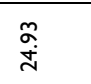 & $\underset{\sim}{\tilde{N}}$ & 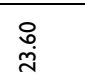 & 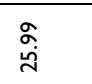 & $\underset{\sim}{\bar{n}}$ & $\stackrel{\frac{\alpha}{z}}{z}$ & $\overline{\tilde{\sim}}$ & $\overline{\tilde{N}}$ & $\stackrel{\tilde{N}}{\tilde{N}}$ & 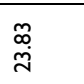 & 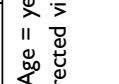 \\
\hline$\underline{0}$ & $\underline{m}$ & \pm & $\underline{m}$ & $\infty$ & $\underline{m}$ & $\underline{n}$ & $\underline{-}$ & $\simeq$ & $\underline{\circ}$ & $\underline{m}$ & \\
\hline 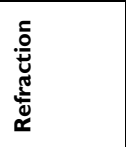 & 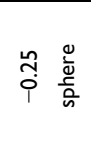 & 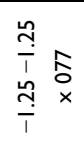 & 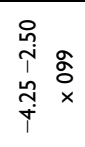 & $\underset{\substack{\stackrel{8}{i} \\
\stackrel{i}{ \pm}}}{\stackrel{t}{x}}$ & $\begin{array}{ll}\hat{n} & \\
\hat{0} & 8 \\
0 & 8 \\
0 & x \\
+ & x\end{array}$ & $\bar{z}$ & 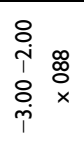 & 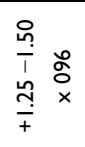 & 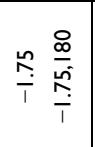 & 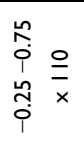 & 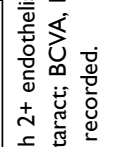 \\
\hline 勇 & 帝 & 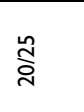 & 京 & 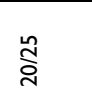 & 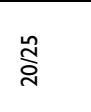 & $\sum_{I}$ & 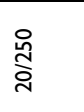 & 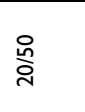 & $\frac{8}{9}$ & 尔 & 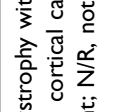 \\
\hline 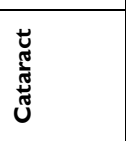 & $\begin{array}{ll} & \\
\tilde{s} & u \\
z & U \\
+ & +\end{array}$ & $\begin{array}{ll}m & \\
s^{*} & U \\
z & U \\
+ & +\end{array}$ & $\begin{array}{l}\tilde{z} \\
+ \\
m\end{array}$ & $\begin{array}{l}气 \\
\pm \\
\pm\end{array}$ & 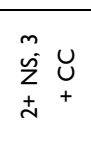 & $\begin{array}{l}u \\
U \\
+\end{array}$ & $\begin{array}{l}\tilde{z} \\
\pm\end{array}$ & $\begin{array}{ll}N & \\
\tilde{s}^{+} & U \\
Z & + \\
+ & +\end{array}$ & $\begin{array}{l}\tilde{z} \\
\pm \\
\pm\end{array}$ & 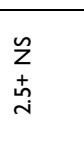 & 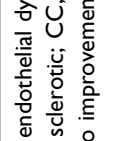 \\
\hline 离 & ő & ०ิ & оิ & ธั & ŏ & о & о & оิ & oิ & оి & \\
\hline ติ & $\Sigma$ & 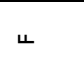 & 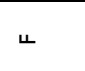 & $\Sigma$ & $\Sigma$ & 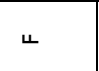 & $\Sigma$ & ч & $\Sigma$ & $\Sigma$ & \\
\hline$\stackrel{8}{\&}$ & $\Phi$ & 8 & ळ & $m$ & $\stackrel{\circ}{\circ}$ & $\pi$ & 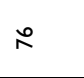 & $\stackrel{\infty}{\curvearrowleft}$ & 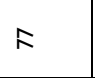 & œ & \\
\hline ฉ & $\underline{n}$ & $\underline{\sim}$ & $\simeq$ & $\underline{\infty}$ & $\underline{a}$ & 요 & $\bar{N}$ & $\pi$ & $\tilde{\sim}$ & $\stackrel{\searrow}{\perp}$ & \\
\hline
\end{tabular}


$92 \%$ had a UDVA of $20 / 100$ or better, and $100 \%$ had a UDVA of 20/200 or better. The largest UDVA improvements manifested in patients presenting with visual acuity of 20/150 or worse, with an average improvement of 0.46 $\log$ MAR in this group. Fourteen patients presented following fragment removal with a best corrected visual acuity (BCVA) of 20/20 or better and 23 with a BCVA of 20/40 or better. One patient had Fuchs endothelial dystrophy with $2+$ endothelial folds.

The mean IOP before cataract extraction (CE) was 14 $\mathrm{mmHg}( \pm 2.5)$ with none higher than $19 \mathrm{mmHg}$. The average IOP measured after $\mathrm{CE}$ but before fragment removal increased by $5 \mathrm{mmHg}(19 \mathrm{mmHg} \pm 8.2)$. The average IOP measured post-fragment removal was $11 \mathrm{mmHg} \pm 2.75$. Statistically significant differences in IOP were measured between pre-CE and time of retained lens fragment diagnosis $(p=0.006)$, time of diagnosis and post lens fragment removal $(\mathrm{p}<0.001)$, and pre-CE and post lens fragment removal $(p<0.001)$. These findings are summarized in Figure 1.

Fifty-eight percent of patients were myopic, with preoperative spherical equivalent ranging from -6.38 to +2.38 D. The mean axial length was $23.82 \mathrm{~mm} \pm 1.11$ (21.3-25.9). Mean anterior chamber depth, aqueous depth, and lens thickness were $3.1 \mathrm{~mm} \pm 0.37,2.58 \mathrm{~mm} \pm 0.31$, and $4.77 \mathrm{~mm} \pm 0.44$, respectively. The mean keratometry value $(\mathrm{Km})$ was $44.01 \mathrm{D} \pm 1.68$ (40.55-47.0) and average white-to -white was $12.11 \mathrm{~mm} \pm 0.46(11.3-13.1)$. Of these, statistically significant differences were measured in ACD ( $\mathrm{p}=$ $0.01)$ and lens thickness $(p=0.001)$ in comparison to the control group. Biometrics are summarized in Table 1 with two-tail $t$-test statistical significance summarized in Table 2.

Fourteen CE surgeries resulting in retained lens fragments were performed using the Horizontal Chop phacoemulsification technique $(58.3 \%)$; the remainder were performed using Divide-and-Conquer. A chi-square test showed no significant difference between surgical techniques $(p=0.71)$. Incidence rate by year is summarized in Figure 2. Yearly incidence rates per surgeon ranged from $0.00 \%$ to $0.85 \%$. Between 2003 and 2021, surgeon 1 had $0.24 \%$ of patients present with retained lens fragments, surgeon 2 had $0.04 \%$, surgeon 3 had $0.14 \%$, surgeon 4 had $0.13 \%$, and surgeon 5 had $0.17 \%$. In 2003 and 2004, surgeon 1 had incidence rates that were significantly higher $(0.31$ and 0.4$)$ compared to the average combined rate of all surgeons throughout the study (0.10), with $\mathrm{p}$ values of 0.001 and 0.003 , respectively.

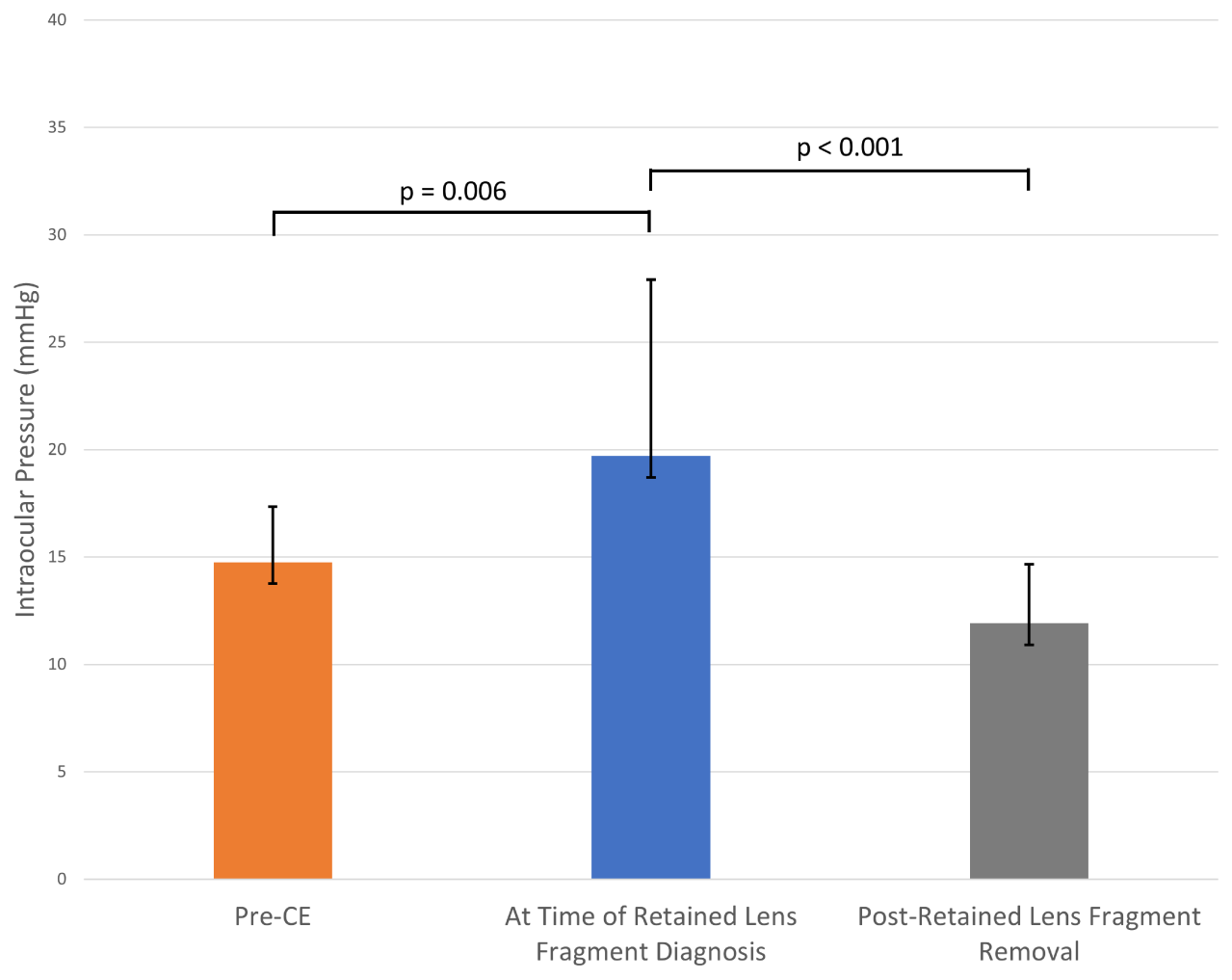

Figure I Mean intraocular pressure measured pre-CE, at the time of retained lens fragment diagnosis, and post-retained lens fragment removal. 
Table 2 Two-Tail t-Test Assuming Equal Variances

\begin{tabular}{|c|c|c|c|c|c|c|c|}
\hline & \multicolumn{2}{|c|}{ Study $(n=24)$} & \multirow[b]{2}{*}{ Range } & \multicolumn{2}{|c|}{ Control $(n=233)$} & \multirow[b]{2}{*}{ Range } & \multirow[t]{2}{*}{ p-value } \\
\hline & Mean & SD & & Mean & SD & & \\
\hline *Age & 76 & \pm 6.72 & $(60-88)$ & 63 & $\pm|I .4|$ & $(22-86)$ & $<0.001$ \\
\hline IOP $(\mathrm{mmHg})$ & 15 & \pm 2.59 & $(8-19)$ & 14 & \pm 2.98 & $(6-26)$ & 0.2 \\
\hline Axial Length (mm) & 23.82 & \pm 1.11 & $(21.31-25.99)$ & 23.88 & \pm 1.28 & $(19.96-28.25)$ & 0.70 \\
\hline *Anterior Chamber Depth (mm) & 3.11 & \pm 0.37 & $(2.37-3.6)$ & 3.33 & \pm 0.39 & $(2.48-4.31)$ & 0.01 \\
\hline Aqueous Depth $(\mathrm{mm})$ & 2.58 & \pm 0.31 & $(2.0-3.04)$ & 2.74 & \pm 0.37 & $(1.94-3.61)$ & 0.06 \\
\hline *Lens Thickness (mm) & 4.77 & \pm 0.44 & $(3.76-5.49)$ & 4.35 & \pm 0.44 & $(3.23-5.34)$ & $<0.001$ \\
\hline $\mathrm{Km}(\mathrm{D})$ & 44.01 & \pm 1.68 & $(40.55-47.0)$ & 43.72 & \pm 3.52 & $(40.25-47.9)$ & 0.69 \\
\hline White-to-White (mm) & 12.11 & \pm 0.46 & $(11.3-13.1)$ & 12.06 & \pm 0.44 & $(10.81-13.24)$ & 0.62 \\
\hline
\end{tabular}

Note: ${ }^{*} p<0.05$

Post-cataract removal clinical findings and management of patients with retained lens fragments can be seen in Table 1. Corneal edema was defined based on a scale of $0-4+{ }^{11}$ all patients presented with some degree of corneal edema ranging from trace to $3.5+$ with a mode of $1+(11$ patients). Four out of 24 patients presented with cells in the anterior chamber (17\%). The majority of retained lens fragments were found in the inferior angle of the anterior chamber (92\%), with the remaining located centrally. The mean number of days between $\mathrm{CE}$ and removal of lens fragments was $26(1-138)$. Ninety-two percent of patients had fragments removed one day following diagnosis, $95 \%$ had fragments removed within two days following diagnosis, and $100 \%$ had fragments removed within three days of diagnosis. Two patients developed cystoid macular edema following fragment removal. These two patients were diagnosed 28 and 35 days after $\mathrm{CE}$. The number of days between CE and fragment removal was not associated with an increased incidence of cystoid macular edema (CME) following fragment removal $(\mathrm{p}=0.8)$.

\section{Discussion}

Retention of lens fragments in the anterior chamber is a rare complication following cataract surgery with phacoemulsification. ${ }^{2}$ Recent studies report incidences of $0.44 \%,{ }^{6}$ although the incidence in this study was $0.10 \%$. Complications of fragment retention range from decreased post-CE visual acuity, corneal edema, increased IOP, and cystoid macular edema, all of which were observed in this study.

Patient sex and laterality were not associated with increased risk of retained lens fragments, however increased age may be associated with higher risk. Patients were also found to have smaller ACDs and thicker

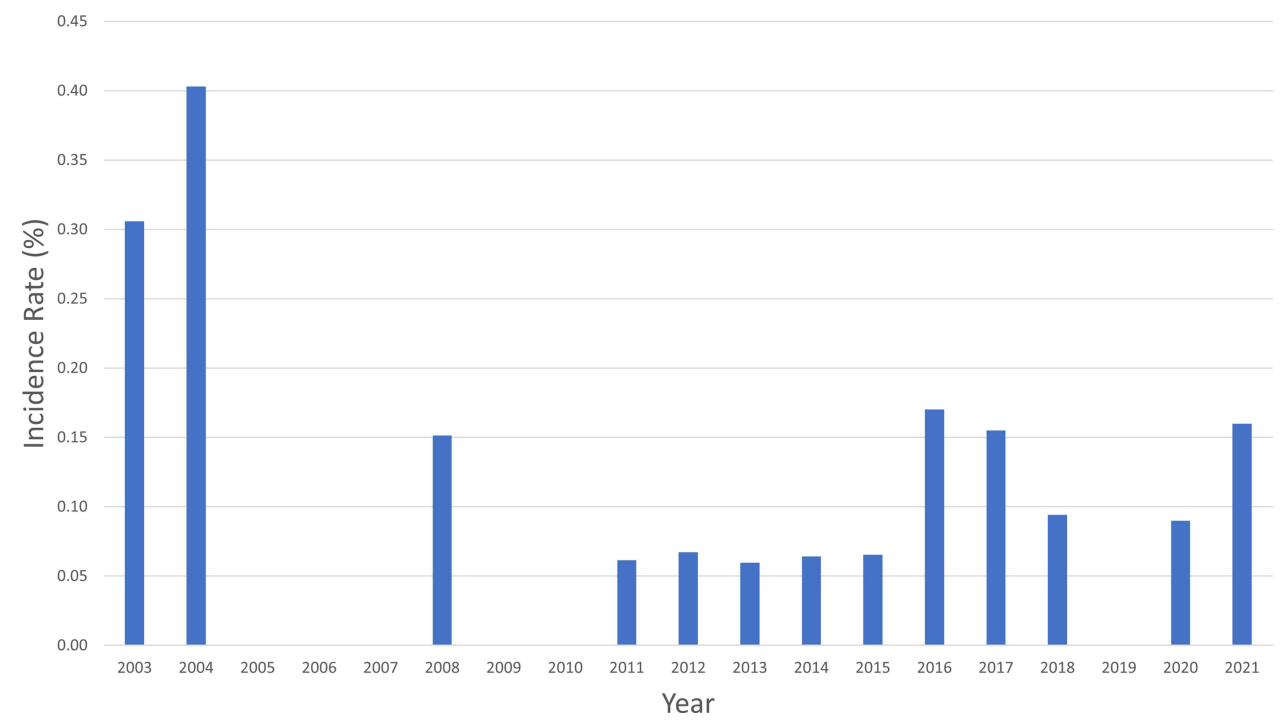

Figure 2 Incidence rate of retained lens fragments following CE per year between 2003 and 2021. 
lenses, compared to the control group. Most patients (14) in the study were myopes, of these, four had a shorter AL than the average AL of the control group (23.88 $\mathrm{mm})$. Myopia in patients with shorter AL may be attributable to a myopic shift associated with nuclear sclerosis. A previous study involving 16 patients reported that larger ACD may be associated with retained lens fragments. ${ }^{8}$ However, the findings in our study are consistent with studies performed by Zavodni et al and Goyal et al reporting increased incidence associated with shallower ACDs. Thicker lenses may predispose patients to retained lens fragments simply due to increased lens volume, resulting in more difficult extraction of all fragments. Surgeons should be especially vigilant of retained fragments during phacoemulsification in patients with thicker lenses. It may be useful for future studies to evaluate the type of fragment (cortical vs nuclear), as some studies have reported worse prognosis associated with nuclear fragments. ${ }^{12}$

The surgeon performing $\mathrm{CE}$ and phacoemulsification technique are two important variables that have not yet been evaluated in recent literature. All five surgeons had more than five years of experience performing lens removal surgeries. Incidence rates over multiple years of practice remained relatively stable among four out of five surgeons, with one surgeon reporting higher rates of retained lens fragment cases during 2003 and 2004. This may indicate that surgeon-specific operating habits and other nonapparent factors may be associated with increased incidence of retained lens fragments. It is also important to note that the decrease in incidence over time throughout this study may be due to improvements in phacoemulsification technologies and more efficient phacodynamics. ${ }^{13}$ Three out of five surgeons used the Divide-and-Conquer phacoemulsification technique while two out of five used the Horizontal Chop, with no statistically significant difference between the two techniques $(p=0.71)$. These findings suggest that while some patient-specific biometric characteristics have proven to be significant risk factors, surgeon-specific operative habits (not including phacoemulsification technique) may also be associated with retained lens fragments in the anterior chamber.

All but two patients in this study presented with symptoms of decreased visual acuity and increased corneal edema despite standard post-CE management. Retained lens fragments were found in the inferior angle of the anterior chamber in $92 \%$ of patients in our study. This is consistent with findings from previous studies $6,8,14$ and may provide an important clue on slit-lamp examination for patients presenting with refractory corneal edema and decreased visual acuity. We recommend that these patients be carefully evaluated for retained lens fragments with special attention given to the inferior angle of the anterior chamber. While all retained lens fragments in this study were visible on slit-lamp examination, previous studies report up to $19 \%$ of cases requiring gonioscopy for visualization and diagnosis of retained lens fragments. ${ }^{6,14,15}$

The average intraocular pressure increased by $5 \mathrm{mmHg}$ upon presentation with retained lens fragments with three patients presenting with IOP $\geq 35 \mathrm{mmHg}$ (Figure 1). This increase in IOP is important to note in patients with existing glaucoma. Lens fragment removal decreased IOP by an average of $8 \mathrm{mmHg}$ and resulted in $100 \%$ of patients with IOP $\leq 18 \mathrm{mmHg}$. While it is possible that these changes in IOP are secondary to retention and removal of viscoelastic, these findings still encourage prompt treatment and removal of retained lens fragments in patients at risk to avoid complications of increased IOP. Two patients in this study developed CME following removal of lens fragments. Goyal et al reported that 3 out of 19 patients developed CME following fragment removal. These findings suggest that the need for repeat surgical intervention or increased ocular inflammation from retained lens fragments may predispose patients to CME.

The average UDVA at the time of fragment discovery was $\log$ MAR 0.49 . Following fragment removal, average UDVA improved to logMAR 0.32. Patients presenting with UDVA $20 / 150$ or worse experienced the most significant improvement in visual outcomes reporting an average improvement of $\log$ MAR 0.56 compared to $\log$ MAR 0.05 in patients with UDVA better than 20/150. These findings support previous data indicating that retained lens fragments decrease visual acuity. ${ }^{6,8,14,16}$ Our study also suggests that patients who present with significant decreases in visual acuity (UDVA worse than 20/150) may benefit the most from lens fragment removal.

There are several limitations concerning our study. It is a retrospective study comprised of a small sample size. Several factors may have impacted our incidence rate. Lack of routine gonioscopy at post-op visits might have resulted in missed subclinical fragments given that our study was based on patients with overt lens fragments that could be easily visualized on slit-lamp examination. Some patients may have had small fragments of retained lens cortex or nucleus and were not symptomatic due to natural resorption of lens material. It is also possible that lens fragments may have migrated into the posterior chamber 
before follow-up exams, resulting in a decreased incidence rate. As phacoemulsification settings were not recorded, we cannot comment on their possible role in the rate of incomplete lens removal. Other limitations include variation in documentation regarding fragment location and type (nuclear vs cortical). There was also lack of documentation of other possible risk factors including floppy iris syndrome, iris color, use of femtosecond laser, and presence of dense corneal arcus or scar limiting visualization during surgery. Surgeon-dependent factors such as case load, fatigue, and operating habits were not analyzed in our paper but may be important variables for future study.

This study shows that increased patient age, small ACDs, and thick lenses may increase the risk of retained lens fragments in the anterior chamber following phacoemulsification. The surgeon performing CE may also present additional surgeon-specific risk factors for retained lens fragments, while phacoemulsification technique (Divideand-Conquer versus Horizontal Chop) showed no significant difference. Patients who present with refractory corneal edema and decreased visual acuity should be evaluated for retained lens fragments, with particular attention examining the inferior angle of the anterior chamber. We recommend that intervention not be delayed once fragments are identified, as this may lead to decreased visual acuity and complications of increased IOP.

\section{Consent}

This case series conforms with the Helsinki Declaration of 1964, as revised in 2013, concerning human and animal rights. The patients signed informed consents for the use of clinical data in research.

\section{Funding}

This study was funded by an unrestricted grant from Research to Prevent Blindness (RPB), 360 Lexington Avenue, 22nd Floor New York, NY 10017. No support was received for the publication of this article.

\section{Disclosure}

All authors declare that they have no conflicts of interest.

\section{References}

1. Wang W, Yan W, Fotis K, et al. Cataract surgical rate and socioeconomics: a global study. Investig Ophthalmol Vis Sci. 2016. doi:10.1167/iovs.16-19894

2. Yorston D. Cataract complications. Community Eye Health J. 2008.

3. Pande M, Dabbs TR. Incidence of lens matter dislocation during phacoemulsification. J Cataract Refract Surg. 1996;22(6):737-742. doi:10.1016/S0886-3350(96)80313-0

4. Kageyama T, Ayaki M, Ogasawara M, Asahiro C, Yaguchi S. Results of vitrectomy performed at the time of phacoemulsification complicated by intravitreal lens fragments. $\mathrm{Br} J$ Ophthalmol. 2001;85 (9):1038-1040. doi:10.1136/bjo.85.9.1038

5. Aasuri MK, Kompella VB, Majji AB. Risk factors for and management of dropped nucleus during phacoemulsification. $J$ Cataract Refract Surg. 2001;27(9):1428-1432. doi:10.1016/S0886-3350(01) 00784-2

6. Norton JC, Goyal S. Patient characteristics and outcomes of retained lens fragments in the anterior chamber after uneventful phacoemulsification. J Cataract Refract Surg. 2018;44(7):848-855. doi:10.1016/j.jcrs.2018.03.034

7. Ho LY, Doft BH, Wang L, Bunker CH. Clinical predictors and outcomes of pars plana vitrectomy for retained lens material after cataract extraction. Am J Ophthalmol. 2009;147(4):587-594.e1. doi:10.1016/j.ajo.2008.10.026

8. Hui JI, Fishler J, Karp CL, Shuler MF, Gedde SJ. Retained nuclear fragments in the anterior chamber after phacoemulsification with an intact posterior capsule. Ophthalmology. 2006;113(11):1949-1953. doi:10.1016/j.ophtha.2006.03.066

9. Ray M, Ho SW, Nga ME. Retained lens fragment in the anterior chamber five years after uncomplicated phacoemulsification with posterior chamber intraocular lens implantation. Indian J Ophthalmol. 2012;60(3):244. doi:10.4103/0301-4738.95891

10. Chylack LT, Leske C, Sperduto R, Khu P, McCarthy D. Lens opacities classification system. Arch Ophthalmol. 1988;106(3):330-334. doi:10.1001/archopht.1988.01060130356020

11. Tandon R, Gupta N, Kalaivani M, Sharma N, Titiyal JS, Vajpayee RB. Amniotic membrane transplantation as an adjunct to medical therapy in acute ocular burns. Br J Ophthalmol. 2011;95 (2):199-204. doi:10.1136/bjo.2009.173716

12. Moisseiev E, Kinori M, Glovinsky Y, Loewenstein A, Moisseiev J, Barak A. Retained lens fragments: nucleus fragments are associated with worse prognosis than cortex or epinucleus fragments. Eur J Ophthalmol. 2011;21(6):741-747. doi:10.5301/EJO.2011.6483

13. Boulter T, Bernhisel A, Mamalis C, et al. Phacoemulsification in review: optimization of cataract removal in an in vitro setting. Surv Ophthalmol. 2019;64(6):868-875. doi:10.1016/j. survophthal.2019.06.007

14. Zavodni ZJ, Meyer JJ, Kim T. Clinical features and outcomes of retained lens fragments in the anterior chamber after phacoemulsification. Am J Ophthalmol. 2015;160(6):1171-1175.e1. doi:10.1016/j.ajo.2015.08.019

15. Gedde SJ, Karp CL, Budenz DL. Retained nuclear fragment in the anterior segment. Arch Ophthalmol. 1998;116(11):1532-1533.

16. Mokhtarzadeh A, Kaufman SC, Koozekanani DD, Meduri A. Delayed presentation of retained nuclear fragment following phacoemulsification cataract extraction. J Cataract Refract Surg. 2014;40 (4):671-674. doi:10.1016/j.jcrs.2014.02.013 


\section{Publish your work in this journal}

Clinical Ophthalmology is an international, peer-reviewed journal covering all subspecialties within ophthalmology. Key topics include: Optometry; Visual science; Pharmacology and drug therapy in eye diseases; Basic Sciences; Primary and Secondary eye care; Patient Safety and Quality of Care Improvements. This journal is indexed on PubMed
Central and CAS, and is the official journal of The Society of Clinical Ophthalmology (SCO). The manuscript management system is completely online and includes a very quick and fair peer-review system, which is all easy to use. Visit http://www.dovepress.com/ testimonials.php to read real quotes from published authors. 